\title{
MIR17HG polymorphisms contribute to High altitude pulmonary edema susceptibility in Chinese Han people
}

\author{
Yanli Zhao \\ Qinghai University \\ Lining Si \\ Affiliated Hospital of Qinghai University \\ Qifu Long \\ Qinghai University \\ Derui Zhu \\ Qinghai University \\ Guoping Shen \\ Qinghai University \\ Rong Wang ( $\square$ rongwang202013@163.com ) \\ Qinghai University
}

\section{Research article}

Keywords: High altitude pulmonary edema, MIR17HG, polymorphism

Posted Date: April 7th, 2020

DOI: https://doi.org/10.21203/rs.3.rs-18553/v1

License: (1) This work is licensed under a Creative Commons Attribution 4.0 International License. Read Full License 


\section{Abstract}

Background: High altitude pulmonary edema (HAPE) is a severe form of acute mountain sickness (AMS). The results of existing studies have shown that the onset of HAPE has obvious ethnic specificity and personal susceptibility, suggesting that the occurrence of HAPE is related to genetic factors. Therefore, six polymorphisms on MIR17HG were selected to investigate the effect of mutations on MIR17HG on HAPE in Chinese Han population.

Materials and Methods: 487 healthy participants (244 participants had high altitude pulmonary edema, as the case group; and 243 participants had no symptoms of HAPE, as the control group) were genotyped via the Agena MassARRAY, and the relationship between polymorphisms on MIR17HG and HAPE risk was evaluated using a $\chi 2$ test with an odds ratio (OR) and $95 \%$ confidence intervals (Cls) in multiple genetic models.

Results: In the allele model, we observed that lower risk (OR $=0.74,95 \% \mathrm{Cl}: 0.56-0.98, \mathrm{p}=0.036)$ of the A allele for rs 7318578 on the MIR17HG compared with the people with the $\mathrm{C}$ allele. Logistic regression analysis of four models for all selected MIR17HG SNPs between cases and controls showed significant differences for rs7318578 (OR = 0.74, 95\%Cl: $0.56-0.98, \mathrm{p}=0.037)$ and rs17735387 (OR = 1.51, 95\%Cl: $1.03-2.21, \mathrm{p}=$ $0.036)$ in the HAPE population.

Conclusion: rs7318578 and rs17735387 on MIR17HG were associated with the genetic susceptibility of HAPE in Chinese Han population.

\section{Introduction}

Among plateau-related diseases, acute mountain sickness (AMS) is the most common. There are regional differences in the incidence of AMS. Generally speaking, the higher the altitude of the plateau, the higher the incidence of AMS and the more severe the clinical symptoms. AMS includes light and heavy types. High altitude pulmonary edema (HAPE) is a heavy type of AMS [1]. It is a serious life-threatening non-cardiogenic pulmonary edema caused by high altitude hypoxic environment. And it is a special disease and common disease in plateau regions, characterized by acute onset, rapid progress and serious consequences. The common altitude of the disease is 2250-4000, which usually occurs within 2 to 5 days after entering the plateau $[1,2]$. The high altitude and low oxygen environment can easily lead to the occurrence of HAPE. More and more evidences proved that genetic factors also play a key role in the occurrence of HAPE. For example, IL1R2[3], NR3C2[4], NR3C1[5], NOS3[6] affect the occurrence of high altitude pulmonary edema.

The pathogenesis of HAPE is more complex, and it is unclear. It is generally believed that the key to its occurrence is the pulmonary vasoconstriction and increased pulmonary arterial pressure caused by hypoxia at high altitude. Studies have found that IncRNA polymorphisms were associated with genetic susceptibility to pulmonary hypertension [7]. Therefore, we believe that IncRNA polymorphisms may also be associated with genetic susceptibility to high altitude pulmonary edema. MIR17HG (known as miR-17-92) was derived from the mir-17-92a-1 cluster host gene, and was a class of pri-miRNAs located in the 800 base pair region of human chromosome 13, whose sole function is to produce six miRNAs (miR-17, miR-18a, miR - 19a, miR $20 a$, miR-19b-1 and miR-92-1) [8,9]. Studies have confirmed that it participates in various growth and development processes such as cell proliferation and differentiation, angiogenesis, and plays an important 
role [10-12]. More and more studies have shown that the polymorphism of MIR17HG gene was associated with breast cancer[13], colorectal cancer[14], Multiple Myeloma[15].

However, the genetic susceptibility to MIR17HG polymorphism and high altitude pulmonary edema is unknown. Therefore, six polymorphisms on MIR17HG were selected to investigate the effect of mutations on MIR17HG on HAPE in Chinese Han population.

\section{Materials And Methods}

\section{Participants}

Based on the strategy of the case-control for explored the gene polymorphisms on MIR17HG gene, we recruited 487 healthy participants from the Affiliated Hospital of Qinghai University. All participants lived in low altitude areas below $2500 \mathrm{~m}$ and reached high altitude areas above $3000 \mathrm{~m}$ due to work or travel. By carrying out symptom (dyspnea), signs (cyanosis at rest) and imaging examination (like X-ray radiograph, computed tomography (CT) of the patient chest) for all participants, and combining with the diagnostic criteria of HAPE [16], found that 244 participants had high altitude pulmonary edema as a case group, while 243 participants had no symptoms of high altitude pulmonary edema, as the control group.

Our present study strictly followed the principles of the Declaration of Helsinki of the World Medical Association and was approved by the Ethics Committee of Qinghai University. Informed consent forms were signed by all participants.

\section{Genotyping}

Combined previous studies, six polymorphisms (rs75267932, rs72640334, rs7336610, rs7318578, rs 17735387, and rs1428) in MIR17HG gene with minor allele frequencies $>5 \%$ in the Chinese Han Beijing population were selected. Using the Agena MassARRAY Assay Design 3.0 Software to design the primer based on the design principles of primer (Table 1). $5 \mathrm{ml}$ of peripheral venous blood was routinely collected in an EDTA-containing anticoagulation tube and stored in an ultra-low temperature freezer for DNA extraction. Extraction of DNA from whole blood samples using the GoldMag-Mini Purification Kit (GoldMag Co. Ltd. Xi'an, China). Quality monitoring of extracted DNA using a spectrophotometer, including concentration and purity. Finally, genotyping of the six gene polymorphisms on MIR17HG gene was performed by the standard protocol from the Agena MassARRAY RS1000 manufacturer, and Agena Typer 4.0 Software was used to analyze the experimental results and obtain genotyping data, which was same as the genotyping method used in previous published articles [3,17].

\section{Statistical Analysis}

The analysis of the general characteristics of the subjects, including Chi-square test was used to compare the difference between the case group and the control group in classified variables (gender). The T-test was used to compare the difference in continuous variables (age) between the case group and the control group. The goodness of fit chi-square test was used to calculate the HWE of each polymorphism in the control group to test the representativeness and randomness of the study population. Chi-square test was used to detect the 
distribution difference of genotype and allele frequency of each polymorphism between the case group and the control group. Logistic regression was used to calculate the OR value and $95 \%$ confidence interval to evaluate the strength of association between each genotype and HAPE by PLINK software. All statistical tests were bilateral, with a significance threshold of 0.05 .

\section{Results}

As table 2 shown that 487 healthy participants were recruited. And 244 participants had high altitude pulmonary edema (female 19, male 225), while 243 participants had no symptoms of high altitude pulmonary edema (female 19, male 224). The mean of age in the HAPE group and healthy group were $32.41 \pm 10.81$ and $32.70 \pm 9.52$ years. No significant differences were found in sex and age between the case and control groups (both $p>0.05$ ).

Table 3 shows that the basic information of six SNPs on the MIR17HG gene in this study. All the SNPs were found to be at Hardy-Weinberg equilibrium based on exact test. In the allele model, we observed that lower risk (OR $=0.74,95 \% \mathrm{Cl}: 0.56-0.98, p=0.036)$ of the A allele for rs7318578 on the MIR17HG gene for HAPE compared with the people with the $C$ allele.

Finally, logistic regression analysis of four models for all selected MIR17HG SNPs between cases and controls showed significant differences for rs7318578 and rs17735387 in the HAPE population. For rs7318578, under the log-additive model, we found that the people carrier the "A" allele decreased the risk of HAPE, when compared with the $\mathrm{C}$ allele carriers, which plays a protective role $(\mathrm{OR}=0.74,95 \% \mathrm{Cl}$ : $0.56-0.98, p=0.037$; Table 4). Individuals carrying the rs 17735387 " $A$ " allele have a $51 \%$ increased risk of HAPE than those with G allele $(\mathrm{OR}=1.51,95 \% \mathrm{Cl}: 1.03-2.21, p=0.036$; Table 4$)$.

\section{Discussion}

Six polymorphisms on MIR17HG were selected to investigate the effect of mutations on MIR17HG on HAPE in Chinese Han population. In our investigative study, we found that MIR17HG rs7318578 minimized the risk and rs17735387 increased the risk.

One research about MIR17HG polymorphism and the risk of breast cancer, revealed that rs 4824505 " $\mathrm{A}$ " allele, rs7336610 "C" allele, and rs4824505/rs7336610 "AC" haplotype were related to the risk of breast cancer, via 244 breast cancer patients and 187 healthy females [13]. In Multiple Myeloma (MM), MIR17HG rs7336610 T allele and MIR17HG rs4284505 G added the risk of MM, while rs7336610-rs4284505 "CA" haplotype decreased the risk of MM. While rs17735387 and rs1428 did not share any relationship with MM risk [15]. In colorectal cancer, rs7336610 and rs 1428 were significantly related to increased risk of colorectal cancer. While, rs7318578 was associated with a decreased risk of colorectal cancer [14]. In our research, we observed that the people carrier the "A" allele decreased the risk of HAPE, when compared with the $\mathrm{C}$ allele carriers; individuals carrying the rs 17735387 " $\mathrm{A}$ " allele have a $51 \%$ increased risk of HAPE than those with $\mathrm{G}$ allele. This is the first time we have found that the MIR17HG mutation was associated with the genetic susceptibility to high altitude pulmonary edema. 
The promoter of the miR-17-92 gene cluster host gene MIR17HG is highly conserved, and the promoter region has multiple conserved transcription factor binding sites [18]. Studies have confirmed that p53 and HIF-1a can inhibit the expression of miR-17-92 gene cluster[19]. And study finds increased expression of HIF-1a is associated with hypoxia adaptation [20]. The MIR17HG gene locus encodes the miR-17-92 gene cluster (MIR17HG), the family members of this gene cluster are widely involved in the functional regulation of vascular ECs [21]. Single-nucleotide polymorphisms (SNPs) could potentially impact biological processes involved in the production or functional effects of the microRNA 17-92 cluster host gene. We found that MIR17HG rs7318578 minimized the risk and rs17735387 increased the risk. Therefore, we speculate that the mutation may affect the expression of MIR17HG in high altitude pulmonary edema, and then affect the occurrence of high altitude pulmonary edema.

Although we found that the MIR17HG mutation was associated with the genetic susceptibility to altitude pulmonary edema. But we need to further verify with large samples. The effects of mutations on the expression of MIR17HG and the molecular mechanisms of MIR17HG in the occurrence of high altitude pulmonary edema were explored through molecular and cell biological methods.

\section{Conclusion}

Through our research, we found that MIR17HG rs7318578 minimized the risk and rs17735387 increased the risk in Chinese Han population, which may be novel biomarkers for susceptibility to HAPE.

\section{Declarations}

\section{Conflict of interest}

All authors declare that they have no conflict of interests.

\section{Acknowledgements}

We thank all participants for taking part in this study. This work was supported by the National Natural Science Foundation of China (No. 81460284, No. 81460051, No. 31560039) and Natural Science Foundation of Qinghai Provincial (No. 2014-ZJ-944Q).

\section{References}

1. Basnyat B, Murdoch DR. High-altitude illness. Lancet. 2003;361(9373):1967-74.

2. Maggiorini M. High altitude-induced pulmonary oedema. Cardiovasc Res. 2006;72(1):41-50.

3. Jin T, Zhu L, Bai M, He X, Wang L, Yuan D, Li S, He Y. Association between the IL1R2 rs2072472 polymorphism and high-altitude pulmonary edema risk. Mol Genet Genomic Med. 2019;7(3):e542.

4. Yang Y, Xu J, Tang F, Ga Q, Li Y, Guan W, Ge RL. NR3C2 Gene is Associated with Susceptibility to HighAltitude Pulmonary Edema in Han Chinese. Wilderness Environ Med. 2018;29(4):488-92.

5. Yang Y, Du H, Li Y, Guan W, Tang F, Ga Q, Ge RL. NR3C1 gene polymorphisms are associated with highaltitude pulmonary edema in Han Chinese. J Physiol Anthropol. 2019;38(1):4. 
6. Wang P, Ha AY, Kidd KK, Koehle MS, Rupert JL. A variant of the endothelial nitric oxide synthase gene (NOS3) associated with AMS susceptibility is less common in the Quechua, a high altitude Native population. High Alt Med Biol. 2010;11(1):27-30.

7. Zhuo Y, Zeng Q, Zhang P, Li G, Xie Q, Cheng Y. Functional polymorphism of IncRNA MALAT1 contributes to pulmonary arterial hypertension susceptibility in Chinese people. Clin Chem Lab Med. 2017;55(1):3846.

8. Zhu H, Han C, Wu T. MiR-17-92 cluster promotes hepatocarcinogenesis. Carcinogenesis. 2015;36(10):1213-22.

9. He L, Thomson JM, Hemann MT, Hernando-Monge E, Mu D, Goodson S, Powers S, Cordon-Cardo C, Lowe SW, Hannon GJ, et al. A microRNA polycistron as a potential human oncogene. Nature. 2005;435(7043):828-33.

10. Lu Y, Thomson JM, Wong HY, Hammond SM, Hogan BL. Transgenic over-expression of the microRNA miR-17-92 cluster promotes proliferation and inhibits differentiation of lung epithelial progenitor cells. Developmental biology. 2007;310(2):442-53.

11. Zhou M, Ma J, Chen S, Chen X, Yu X. MicroRNA-17-92 cluster regulates osteoblast proliferation and differentiation. Endocrine. 2014;45(2):302-10.

12. Dews M, Fox JL, Hultine S, Sundaram P, Wang W, Liu YY, Furth E, Enders GH, El-Deiry W, Schelter JM, et al. The myc-miR-17 92 axis blunts TGF signaling and production of multiple TGF-dependent antiangiogenic factors. Cancer Res. 2010;70(20):8233-46.

13. Chacon-Cortes D, Smith RA, Lea RA, Youl PH, Griffiths LR. Association of microRNA 17-92 cluster host gene (MIR17HG) polymorphisms with breast cancer. Tumour Biol. 2015;36(7):5369-76.

14. Chen P, Bai Y, Li Y, Yuan Y, Cheng Y, Pang J, Zhu H, Chen C. Association between polymorphisms of MIR17HG and risk of colorectal cancer in the Chinese Han population. Mol Genet Genomic Med. 2019;7(6):e667.

15. Wu H, Huang T, Ye Z, Fu X, Hu K, Yang X. Correlation of MicroRNA 17-92 Cluster Host Gene (MIR17HG) Polymorphisms With Susceptibility and Prognosis for Multiple Myeloma. Clin Lymphoma Myeloma Leuk. 2019;19(7):e359-66.

16. Gabry AL, Ledoux X, Mozziconacci M, Martin C. High-altitude pulmonary edema at moderate altitude (< $2,400 \mathrm{~m} ; 7,870$ feet): a series of 52 patients. Chest. 2003;123(1):49-53.

17. He X, Zheng J, He Y, Wang Y, Wang L, Bai M, Jin T, Yuan D. Long Non-coding RNA LINC-PINT and LINC00599 Polymorphisms are Associated With High-altitude Pulmonary Edema in Chinese. Arch Bronconeumol 2019.

18. Ji M, Rao E, Ramachandrareddy H, Shen Y, Jiang C, Chen J, Hu Y, Rizzino A, Chan WC, Fu K, et al. The miR17-92 microRNA cluster is regulated by multiple mechanisms in B-cell malignancies. Am J Pathol. 2011;179(4):1645-56.

19. Yan HL, Xue G, Mei Q, Wang YZ, Ding FX, Liu MF, Lu MH, Tang Y, Yu HY, Sun SH. Repression of the miR17-92 cluster by $\mathrm{p} 53$ has an important function in hypoxia-induced apoptosis. EMBO J. 2009;28(18):2719-32. 
20. Tsai SH, Huang PH, Tsai HY, Hsu YJ, Chen YW, Wang JC, Chen YH, Lin SJ. Roles of the hypoximir microRNA-424/322 in acute hypoxia and hypoxia-induced pulmonary vascular leakage. FASEB J. 2019;33(11):12565-75.

21. Chamorro-Jorganes A, Lee MY, Araldi E, Landskroner-Eiger S, Fernandez-Fuertes M, Sahraei M, Quiles Del Rey M, van Solingen C, Yu J, Fernandez-Hernando C, et al. VEGF-Induced Expression of miR-17-92 Cluster in Endothelial Cells Is Mediated by ERK/ELK1 Activation and Regulates Angiogenesis. Circ Res. 2016;118(1):38-47.

\section{Tables}

Table 1 Primer Sequences of MIR17HG Gene SNPs

\begin{tabular}{|c|c|c|c|}
\hline SNP_ID & 2nd-PCRP & 1st-PCRP & UEP_SEQ \\
\hline rs75267932 & ACGTTGGATGTTAGAGAGAATGCCGCTCTG & ACGTTGGATGCCCAACCCTAAATTCCATGC & сcсCCGCTCTGTTTAAAGCAATGTGTA \\
\hline rs72640334 & ACGTTGGATGGCTTAAGAACTCTGCTAATG & ACGTTGGATGCTATCATTCTGGAGTTGATG & САСТGTTCATTTCACATCСТ \\
\hline rs7336610 & ACGTTGGATGACAGCGTTTCACCATGTCGG & ACGTTGGATGAAAAAGTTCCGGCTGGACAC & CTCCTGACCTCAGGTAATCC \\
\hline rs7318578 & ACGTTGGATGGAAATCATCCAGCAGGCTTC & ACGTTGGATGCAGCATGGTCTGGTAGTTTG & TCCTATCACACTGTTCCA \\
\hline rs17735387 & ACGTTGGATGGCTTTCTTTCCAAATATAGGC & ACGTTGGATGAGCCTTAACTATTTGGAGGG & AATAGAAAGTTGTACATGCAAA \\
\hline rs1428 & ACGTTGGATGTCAATATTCTCGTTCTGGAC & ACGTTGGATGTGGCTGTTTGAGTTCTAGCG & tACAATTTCTTAACAGCTTTAAAAT \\
\hline
\end{tabular}

Table 2 Characteristics of participants

\begin{tabular}{cccc}
\hline Variables & case (244) & control (243) & $p$ \\
\hline Age \pm SD & $32.41 \pm 10.81$ & $32.70 \pm 9.52$ & 0.761 \\
Sex & & & 0.869 \\
Female & 19 & 19 & \\
male & 225 & 224 & \\
& & & \\
\hline
\end{tabular}

$\mathrm{p}<0.05$ indicates statistical significance

Table 3 Basic information of six SNPs on the MIR17HG gene in this study 


\begin{tabular}{|c|c|c|c|c|c|c|c|c|c|}
\hline SNP & Chromosome & $\mathrm{BP}$ & Alleles & Gene & MAF-Case & MAF-Control & HWE-p & OR $(95 \% \mathrm{CI})$ & $p$ \\
\hline rs75267932 & 13 & 91351812 & $\mathrm{~A} / \mathrm{G}$ & MIR17HG & 0.125 & 0.115 & 0.750 & $1.10(0.75-1.62)$ & 0.639 \\
\hline rs72640334 & 13 & 91352674 & $\mathrm{~A} / \mathrm{C}$ & MIR17HG & 0.102 & 0.103 & 0.726 & $1.00(0.66-1.51)$ & 0.983 \\
\hline rs7336610 & 13 & 91352883 & $\mathrm{C} / \mathrm{T}$ & MIR17HG & 0.471 & 0.484 & 0.608 & $0.95(0.74-1.22)$ & 0.703 \\
\hline rs7318578 & 13 & 91353215 & $\mathrm{~A} / \mathrm{C}$ & MIR17HG & 0.256 & 0.317 & 0.882 & $0.74(0.56-0.98)$ & 0.036 \\
\hline rs17735387 & 13 & 91353800 & $\mathrm{~A} / \mathrm{G}$ & MIR17HG & 0.199 & 0.160 & 0.229 & $1.30(0.93-1.80)$ & 0.120 \\
\hline rs1428 & 13 & 91354516 & $\mathrm{G} / \mathrm{T}$ & MIR17HG & 0.475 & 0.484 & 0.608 & $0.97(0.75-1.25)$ & 0.800 \\
\hline
\end{tabular}

HWE, Hardy-Weinberg Equilibrium; ORs, odds ratios; CI: confidence interval. $\mathrm{p}<0.05$ indicates statistical significance

Table 4 Correlation of MIR17HG SNPs with HAPE risk

\begin{tabular}{|c|c|c|c|c|c|c|c|c|c|}
\hline \multirow[t]{2}{*}{ SNP } & \multirow[t]{2}{*}{ Model } & \multirow[t]{2}{*}{ Genotype } & \multicolumn{2}{|r|}{ Number } & \multicolumn{2}{|c|}{ Crude analysis } & \multicolumn{2}{|c|}{ adjusted by age and sex } & \multirow{2}{*}{$\begin{array}{l}\text { ORs, } \\
\text { odds }\end{array}$} \\
\hline & & & Case & Control & OR $(95 \% \mathrm{CI})$ & $p$ & OR $(95 \% \mathrm{CI})$ & $p$ & \\
\hline \multirow[t]{6}{*}{ rs7318578 } & Genotype & $\mathrm{CC}$ & 134 & 114 & 1 & & 1 & & \\
\hline & & $\mathrm{AC}$ & 95 & 104 & $0.78(0.53-1.13)$ & 0.186 & $0.78(0.54-1.14)$ & 0.198 & \\
\hline & & AA & 15 & 25 & $0.51(0.26-1.02)$ & 0.055 & $0.51(0.26-1.01)$ & 0.053 & \\
\hline & dominant & $\mathrm{CC} / \mathrm{AC}+\mathrm{AA}$ & $134 / 110$ & $114 / 129$ & $0.73(0.51-1.04)$ & 0.078 & $0.73(0.51-1.04)$ & 0.081 & \\
\hline & recessive & $\mathrm{CC}+\mathrm{AC} / \mathrm{AA}$ & $229 / 15$ & $228 / 25$ & $0.57(0.29-1.11)$ & 0.100 & $0.57(0.29-1.10)$ & 0.094 & \\
\hline & log-additive & l & l & 1 & $0.74(0.56-0.98)$ & 0.037 & $0.74(0.56-0.98)$ & 0.037 & \\
\hline \multirow[t]{3}{*}{ rs17735387 } & Genotype & GG & 153 & 174 & 1 & & 1 & & \\
\hline & & AG & 85 & 60 & $1.61(1.09-2.39)$ & 0.018 & $1.62(1.09-2.41)$ & 0.018 & \\
\hline & & AA & 6 & 9 & $0.76(0.26-2.18)$ & 0.607 & $0.76(0.27-2.20)$ & 0.617 & \\
\hline \multirow[t]{3}{*}{ dominant } & $\mathrm{GG} / \mathrm{AG}+\mathrm{AA}$ & $153 / 91$ & $174 / 69$ & $1.50(1.03-2.20)$ & 0.037 & $1.51(1.03-2.21)$ & 0.036 & & \\
\hline & recessive & $\mathrm{GG}+\mathrm{AG} / \mathrm{AA}$ & $238 / 6$ & $234 / 9$ & $0.66(0.23-1.87)$ & 0.430 & $0.66(0.23-1.88)$ & 0.434 & \\
\hline & log-additive & 1 & 1 & 1 & $1.30(0.93-1.81)$ & 0.119 & $1.31(0.94-1.82)$ & 0.117 & \\
\hline
\end{tabular}

confidence interval. $\mathrm{p}<0.05$ indicates statistical significance 